\title{
Re-irradiation or re-operation followed by dendritic cell vaccination? Comparison of two different salvage strategies for relapsed high-grade gliomas by means of a new prognostic model
}

\author{
Klaus Müller ${ }^{1,2}$ - Guido Henke ${ }^{3,4}$ - Sophie Pietschmann ${ }^{1} \cdot$ Stefaan van Gool $^{5}$. \\ Steven De Vleeschouwer ${ }^{5}$. André O. von Bueren ${ }^{6} \cdot$ Inge Compter $^{7} \cdot$ \\ Carsten Friedrich $^{8}$ - Christiane Matuschek ${ }^{9}$ Gunther Klautke ${ }^{2}$. \\ Rolf-Dieter Kortmann ${ }^{1}$. Thomas Hundsberger ${ }^{10}$ - Brigitta G. Baumert ${ }^{7,11}$
}

Received: 9 January 2015/ Accepted: 8 June 2015

(C) Springer Science+Business Media New York 2015

\begin{abstract}
We aimed to compare two different salvage treatment strategies for relapsed high-grade glioma (HGG) patients by means of a new prognostic model. A simplified version of the so-called HGG-Immuno RPA model estimates the prognosis of relapsed HGG patients and distinguishes three different prognostic classes $(\mathrm{I}=$ good, II = intermediate, III = poor). The model has been constructed with a cohort of 117 patients whose salvage treatment consisted of re-operation followed by dendritic cell vaccination (ReOP $+\mathrm{DCV})$. However, using only the predictors histology, age and performance status, the simplified HGG-Immuno RPA model is basically independent from treatment. In the present study we applied the simplified model to the cohort used to construct the original
\end{abstract}

Thomas Hundsberger and Brigitta G. Baumert have contributed equally to this article.

Klaus Müller

klausmueller1978@googlemail.com

1 Department of Radiation Oncology, University of Leipzig Medical Center, Leipzig, Germany

2 Department of Radiation Oncology, Hospital Chemnitz, Bürgerstraße 2, 09113 Chemnitz, Germany

3 Department of Radiation Oncology, Cantonal Hospital St. Gallen, St. Gallen, Switzerland

4 Department of Radiation Oncology, University Hospital Tübingen, Tübingen, Germany

5 Laboratory of Experimental Immunology, Catholic University Leuven, Leuven, Belgium

6 Division of Pediatric Hematology and Oncology, Department of Pediatrics and Adolescent Medicine, University Medical Center Göttingen, Göttingen, Germany
HGG-Immuno RPA model and another cohort of 165 patients who underwent re-irradiation (ReRT) at relapse. Then, we compared the outcomes achieved by the two different salvage treatments in each prognostic class. The model predicted good, intermediate and poor prognosis for 11, 31 and 75 patients of the ReOP + DCV cohort and for 20, 39 and 106 patients of the ReRT cohort, respectively. Neither of the two strategies was superior to the other. In the groups with good, intermediate and poor prognosis 12-months survival rates were 73,59 and $25 \%$ after $\mathrm{ReOP}+\mathrm{DCV}$ and 72,36 and $23 \%$ after ReRT, respectively. Being easy to handle and independent from treatment, the aforementioned model is useful for therapeutic decisions. ReRT and ReOP + DVC seem to be equally effective. The choice of salvage treatment should be based on the expected side effects.

7 Department of Radiation Oncology (MAASTRO), GROW (School for Oncology \& Developmental Biology), University of Maastricht Medical Center, Maastricht, The Netherlands

8 Division of Pediatric Oncology, Hematology and Hemostaseology, Department of Woman's and Children's Health, University Hospital Leipzig, Leipzig, Germany

9 Department of Radiation Oncology, Medical Faculty, Heinrich Heine University of Düsseldorf, Düsseldorf, Germany

10 Departments of Neurology and Haematology/Oncology, Cantonal Hospital St. Gallen, St. Gallen, Switzerland

11 Clinical Cooperation Unit Neurooncology, Department of Radiation Oncology, MediClin Robert Janker Clinic \& University of Bonn Medical Center, Bonn, Germany 
Keywords Relapsed high-grade gliomas - Prognostic model $\cdot$ Re-irradiation - Dendritic cell vaccination . Recurrent glioblastoma $\cdot$ Salvage treatment $\cdot$ Prognosis

\section{Background}

The best treatment approach for relapsed high-grade gliomas (HGG) is currently unknown. Randomized trials on the topic are sparse. Thus, for clinical decision making physicians mostly have to rely on experiences from small retrospective series [1-6]. Prognostic models for post-relapse survival may be beneficial for the rational choice of salvage treatment since they may enhance the comparability of heterogeneous patient cohorts by defining homogeneous subsets of patients with similar intrinsic prognosis. Within these subsets more accurate comparisons of different salvage strategies may be drawn. Recently, a simple recursive partitioning analysis (RPA) model for the survival of relapsed HGG patients undergoing re-operation followed by dendritic cell vaccination $(\mathrm{ReOP}+\mathrm{DCV})$ was suggested. The so-called HGG-Immuno RPA model distinguished four prognostic classes (

$\mathrm{I}=$ excellent,$\quad \mathrm{II}=$ good,$\quad \mathrm{III}=$ intermediate,$\quad \mathrm{IV}=$ poor), which were characterized by different survival rates [7].

The purpose of the present study was:

(1) To validate the reproducibility of a simplified version of the HGG-Immuno RPA model in terms of discrimination with an independent patient cohort.

(2) To compare two different salvage treatments: reirradiation (ReRT) versus re-operation followed by dendritic cell vaccination ( $\mathrm{ReOP}+\mathrm{DCV})$ by means of the model.

\section{Patients and methods}

\section{The HGG-Immuno RPA model}

Recursive partitioning analysis is a statistical technique which is used to define prognostic classes based on treatment and/or pre-treatment prognostic variables (predictors) [8]. The HGG-Immuno RPA model was recently suggested for the prognostic classification of relapsed HGG patients undergoing re-operation followed by dendritic cell vaccination [7]. The original model was constructed with a cohort of 117 adult patients included in the early phase of the HGG-Immuno-2003 trial (ReOP + DCV cohort) [9]. It distinguishes four prognostic classes ( $\mathrm{I}=$ excellent, $\mathrm{II}=$ good, III = intermediate, IV = poor) and is based on four predictors (age, WHO grading, performance status and mental status) [7].
For this study, however, we used a simplified version of the model in which the predictor "mental status" was omitted. As this parameter is only decisive in the prognostic classes III and IV of the original model, these classes were merged together forming a joint poor prognosis group (“class III") (Fig. 1).

\section{Patient cohorts}

We applied the simplified version with three different prognostic classes $(\mathrm{I}=$ good, $\mathrm{II}=$ intermediate, $\mathrm{III}=$ poor) to

(a) the patient cohort used to construct the original model ("ReOP + DCV cohort") and

(b) an independent, multicenter cohort of 165 relapsed HGG patients who underwent a second course of radiation therapy (ReRT) instead of $\mathrm{ReOP}+\mathrm{DCV}$ ("ReRT cohort").

Detailed information on patient characteristics, treatment and outcome of the ReOP + DCV cohort has already been published [7]. Survival time was defined as the time

(a) original HGG-Immuno RPA model:

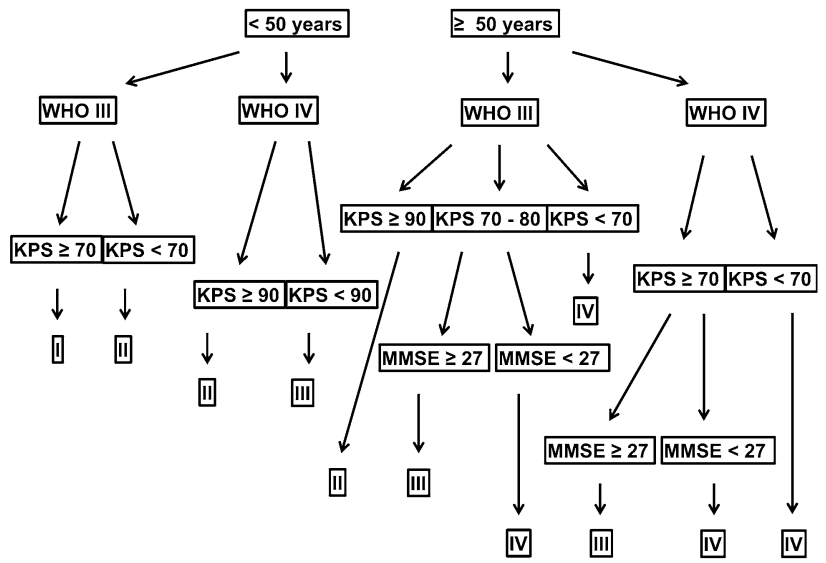

(b) simplified HGG-Immuno RPA model:

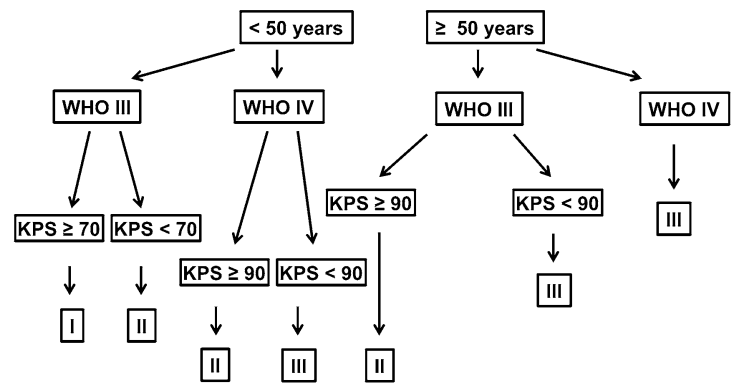

Fig. 12 HGG-IMMUNO recursive partitioning analysis (RPA) models to predict the prognosis of relapsed HGG patients. a Original HGG-IMMUNO RPA tree in relapsed high-grade glioma (HGG) patients as suggested by Vleeschouwer et al. [7]. b Simplified version of the aforementioned model which may be used whenever mini mental state examination was not performed 
from pre-vaccine re-operation to death or to last follow-up. In the ReRT cohort follow-up time of survivors $(\mathrm{n}=32)$ ranged from 0.6 to 69.1 months (median, 8.5 months). For this cohort, survival time was defined as the time from the start of re-irradiation to death or to last follow-up. The dataset was collected from four different hospitals, namely the University Hospital of Leipzig (Germany) $(n=64$ patients), the University Hospital of Tübingen (Germany) $(\mathrm{n}=31$ patients), the Cantonal Hospital of St. Gallen (Switzerland) ( $\mathrm{n}=13$ patients) and the University Hospital of Maastricht (The Netherlands) ( $n=57$ patients). Characteristics and outcome of the patients from Leipzig, Tübingen and St. Gallen have already been published [1012]. Of note, a significant proportion of the re-irradiated patients also underwent ReOP.

\section{Working assumption}

As the predictors of the simplified model (age, histology and performance status) are not related to therapy, we set up the hypothesis that it would keep its ability to discriminate patients with different prognoses in both cohorts or, in other words, independently of the treatment strategy used. Moreover, we postulated that if one salvage treatment was more efficient than the other in terms of post-treatment survival calibration of the model would differ between both cohorts.

\section{Methodological approach}

The prognostic model was validated with a patient cohort independent from the one used to construct it. We addressed the two fundamental aspects of a prognostic model's performance: discrimination and calibration. Discrimination reflects the ability of a model to characterize subsets of patients with different prognoses. Calibration usually reflects prediction accuracy $[13,14]$. However, in this study, calibration reflects the extent to which the antitumoral potentials of the two different salvage treatments, $\mathrm{ReRT}$ and ReOP + DCV, match each other.

Our analysis included the following four steps:

(1) In both cohorts discrimination and calibration of the RPA model were roughly checked by visual comparison of the survival curves corresponding to the three RPA classes (Fig. 2).

(2) In both cohorts discrimination was assessed calculating hazard ratios for deaths (Cox regression analysis) for the RPA classes I and II and plotting them in the form of a tree. The worst prognostic class III served as reference (Fig. 3).

(3) For each RPA class calibration was separately checked by visual comparison of the Kaplan-Meier survival curve in the re-irradiation cohort with its counterpart in the vaccination cohort and the corresponding $95 \%$ confidence intervals (CIs) (Fig. 4).

(4) By means of calibration plots the 9-, 12- and 15-months survival probabilities of the vaccinated patients (X-axis) were related to the Kaplan-Meier estimates of the re-irradiated patients (Y-axis). In such a calibration plot Kaplan-Meier estimates close to the bisecting line indicate that the two compared treatments are equally effective (Fig. 5). (a) Vaccination cohort

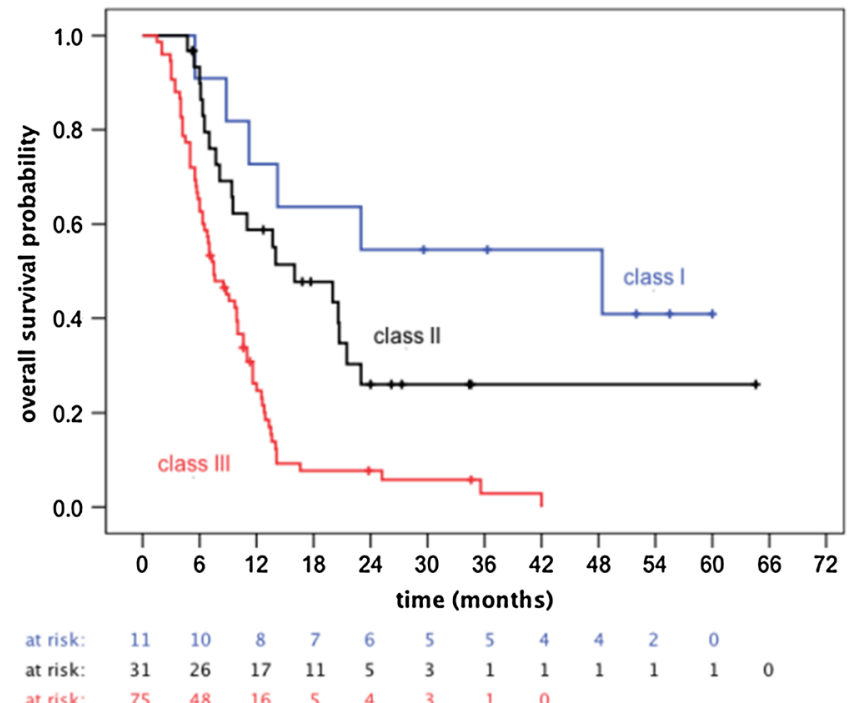

(b) Re-irradiation cohort

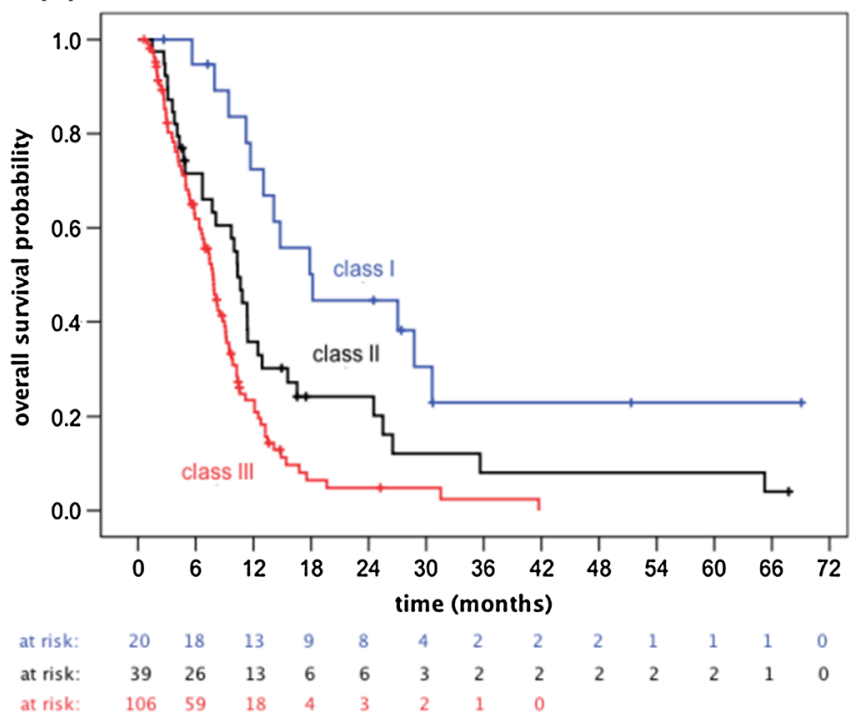

Fig. 2 Kaplan-Meier survival plots according to the three different RPA classes in a the vaccination (ReOP + DCV) cohort and $\mathbf{b}$ the reirradiation (ReRT) cohort 


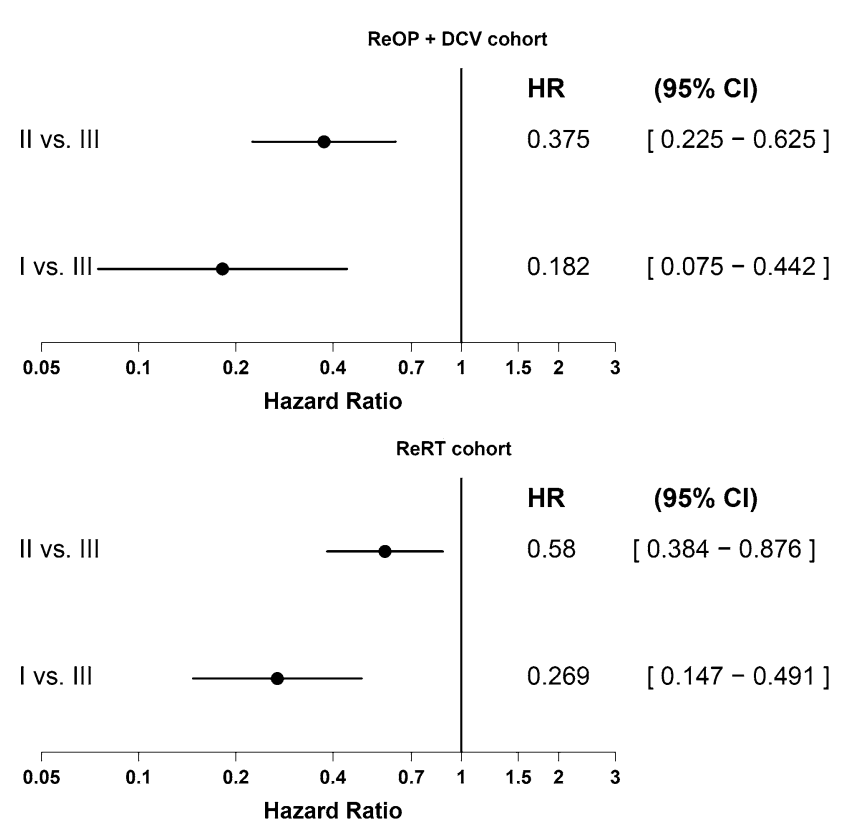

Fig. 3 Tree plot of the hazard ratios for deaths according to RPA classes. Hazard ratios for deaths of RPA classes I and II in the vaccination $(\mathrm{ReOP}+\mathrm{DCV})$ cohort (above) and in the re-irradiation (ReRT) cohort (below). RPA class III served as reference

The follow-up time of the patients was quantified according to the method suggested by Schemper and Smith [15].

The statistical computations of the follow-up time and (1) were performed with SPSS, version 20.0. The statistical computations of (2), (3) and (4) were performed with R, version 3.0.3.

\section{Results}

\section{Baseline patient characteristics and outcomes}

The characteristics of both treatment cohorts $(\mathrm{ReOP}+\mathrm{DCV}$ versus ReRT) are shown in Table 1.

In the ReOP + DCV cohort 95 patients $(81.2 \%)$ died during the period of observation. Median follow-up time was $34.6 \pm 3.5$ months. Median survival times after $\mathrm{ReOP}+\mathrm{DCV}$ were 48.4, 16.0 and 7.5 months in the classes with good, intermediate and poor prognosis, respectively.

In the ReRT cohort 133 patients $(80.6 \%)$ died during the period of observation. Median follow-up time was $30.7 \pm 10.9$ months. Median survival times after ReRT were 18.2, 10.3 and 7.8 months in the classes with good, intermediate and poor prognosis, respectively.

The 6- and 12-month overall survival rates of the different prognostic classes sorted by treatment $(\mathrm{ReOP}+\mathrm{DCV}$ versus ReRT) are listed in Table 2.
Step 1: visual comparison

Figure 2a shows the Kaplan-Meier survival curves of the 117 patients who underwent $\mathrm{ReOP}+\mathrm{DCV}$ according to the three prognostic classes of the simplified HGG-Immuno RPA model. Figure $2 b$ shows the corresponding curves of the 165 re-irradiated patients. Both in Fig. $2 a$ and $b$ the survival curves of the three different prognostic classes I, II and III separate nicely from each other-a finding which suggests the validity of the simplified RPA model.

Step 2: quantifying discrimination of the simplified $H G G$ Immuno RPA model in both treatment cohorts

Using both the vaccination cohort and the re-irradiation cohort, we calculated the hazard ratios for death (HR) of the prognostic classes I and II. The worst prognostic class (III) served as reference (Fig. 3). The HR can be interpreted as the chance of death occurring in the corresponding prognostic class divided by the chance of death occurring in the worst prognostic class. Ideally, the HR for the corresponding risk classes should satisfy two criteria. First, the HR should increase steadily and clearly as the risk class increases, indicating a higher hazard of death in the more unfavorable prognostic classes. Second, the HR should remain clearly below a value of 1.0 , as class III has the worst prognosis by definition. Both criteria are fulfilled in both treatment cohorts (Fig. 3).

Step 3: assessing treatment benefit by a comparison of survival plots sorted by RPA class

For each RPA class the survival plot of re-irradiated patients (colored) was compared with the survival plot of vaccinated patients (black) and its corresponding $95 \%$ confidence intervals (black, dashed). Colored curves located between the two black-dashed lines suggest that ReRT and $\mathrm{ReOP}+\mathrm{DCV}$ are similar with regards to effectivity. This criterion is best fulfilled in RPA class III i.e. in the patients with the worst prognosis. For class I the $95 \% \mathrm{CI}$ is extremely wide due to the small sample size, a fact, certainly reducing the meaningfulness of the analysis (Figure 4).

Step 4: comparing treatment benefit by means of "calibration curves" for the time points 9, 12 and 15 months after the start of salvage treatment

Figure 5 demonstrates the calibration curves sorted by RPA class. The 9-, 12- and 15 months survival rates of the different RPA classes in the ReRT cohort (Y-axis) are compared with the corresponding survival rates in the $\mathrm{ReOP}+\mathrm{DCV}$ cohort (X-axis). The colored vertical lines 

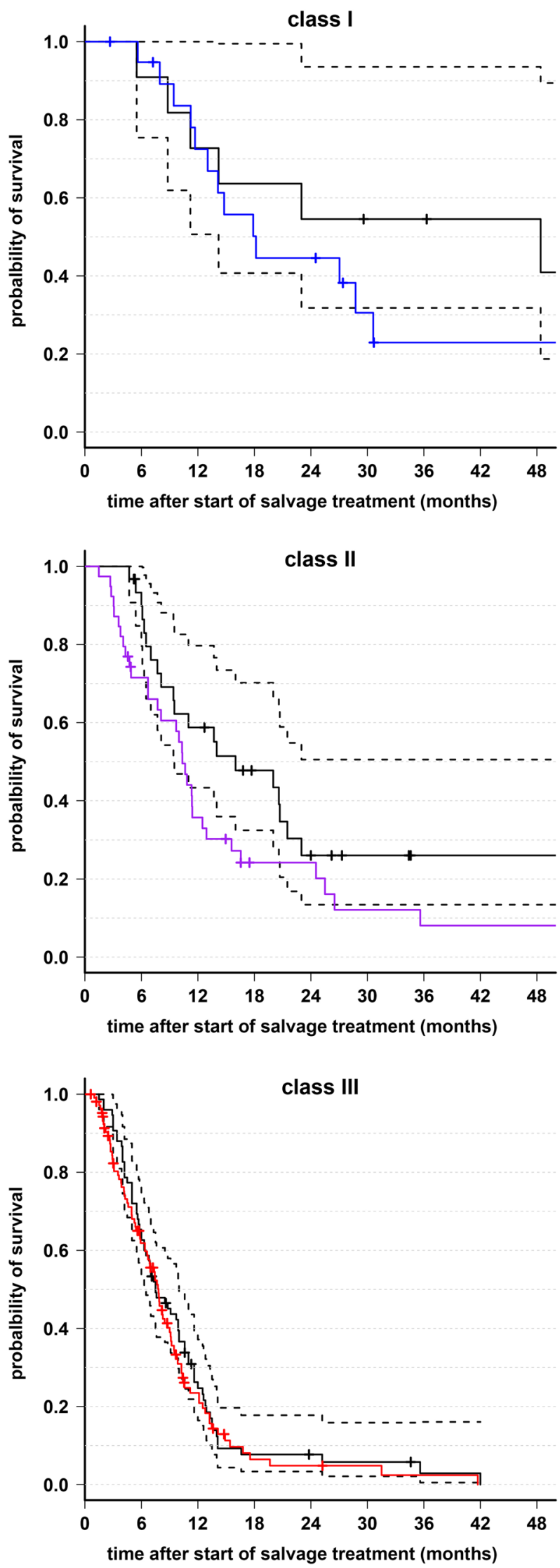

4Fig. 4 Comparison of survival plots sorted by RPA class. Survival plot of the ReRT cohort (coloured) compared with the survival plot of the $\mathrm{ReOP}+\mathrm{DCV}$ cohort (black) and its corresponding $95 \%$ confidence interval (back, dashed) for the RPA-classes I-III

represent the $95 \%$ confidence intervals of the respective survival rates in the ReRT cohort. Vertical lines crossing the bisecting line indicate that ReRT and $\mathrm{ReOP}+\mathrm{DCV}$ are equally effective. This criterion is best fulfilled in RPA class III i.e. in the patients with the worst prognosis (Fig. 5).

\section{Discussion}

The treatment of newly diagnosed Glioblastoma is highly standardized and currently comprises surgery followed by radiation therapy plus concomitant and adjuvant temozolomide. In anaplastic gliomas molecular markers like 1p/ $19 \mathrm{q}$ codeletion and IDH mutations will strongly guide treatment [16]. In contrast, the optimal salvage treatment approach after relapse has not been determined yet and prospective, randomized trials are sparse. Although a plethora of mono- and combination chemotherapy strategies have been evaluated, an obvious survival benefit has not been achieved with any particular regimen to date [17]. The role of re-operation is controversially discussed. Some authors observed that second surgery was of limited value for survival if complete resection could not be achieved. Accordingly, they recommended cytoreductive therapy only in symptomatic patients $[4,5]$. Others argued that reoperation might be associated with considerable morbidity and mortality [18]. In contrast, there is also a study claiming that repeated (incomplete) resections may result in improved survival and that the risk of iatrogenic deficits caused by repeated surgery is low [19]. Postoperative dendritic cell (DC) vaccination is a relatively new and potentially promising approach exploiting the stimulation of the host immune system against tumor antigens [20]. Adjuvant DC based immunotherapy is safe and feasible for a considerable number of patients. However, the extent of resection at the start of vaccination is critical for success [7, $9,21]$. Re-irradiation has been widely accepted as a salvage therapy in recurrent gliomas with stereotactic fractionated radiotherapy being the most frequent technique. Toxicity has been overestimated for a long time [6, 18]. The antitumoral effect of re-irradiation may be enhanced by radiosensitizing agents. For instance, the results of the prospective, randomized APG101 study indicate a benefit of combining re-irradiation with CD95 pathway inhibition [22]. The National Comprehensive Cancer Network and Canadian $(\mathrm{NCCN})$ guidelines for recurrent or progressive 

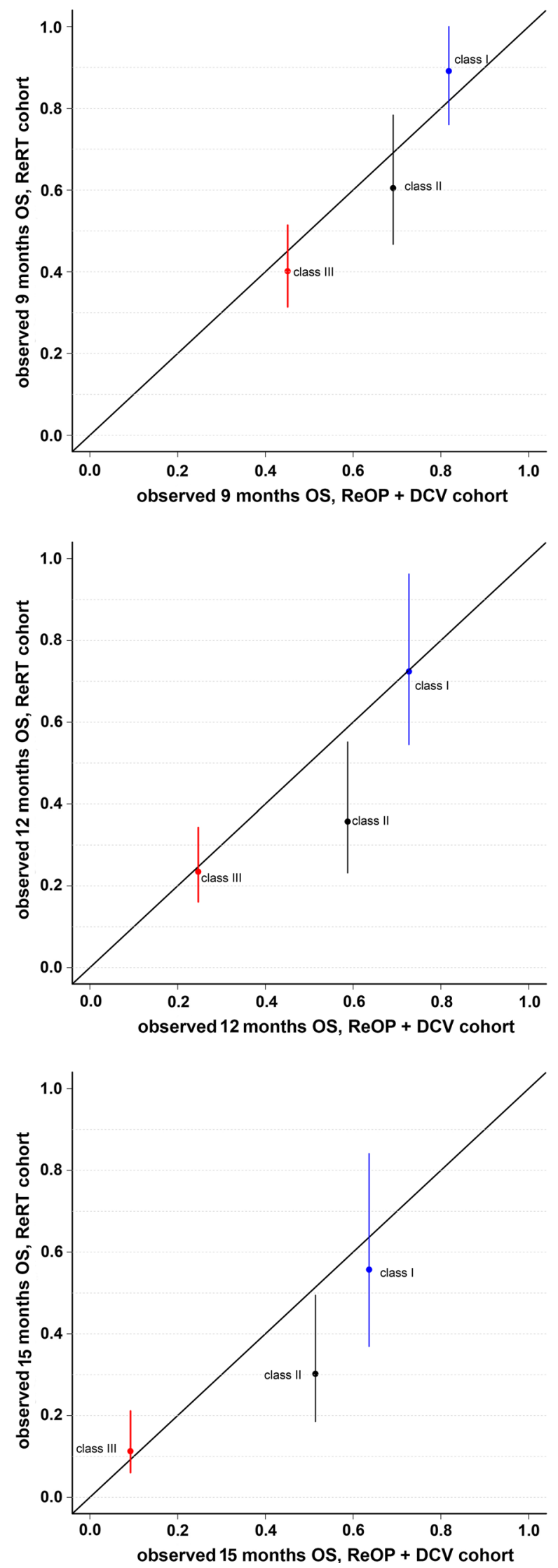

4Fig. 5 Calibration plots. Referring to certain time points $(9,12$ and 15 months after the start of salvage treatment), the calibration plots compare the survival rates of the vaccinated patients sorted by the different RPA classes ( $X$-axis) with the respective survival rates and their $95 \%$ confidence intervals of the re-irradiated patients ( $Y$-axis). Kaplan-Meier estimates close to the bisecting line indicate that reirradiation and re-operation followed by adjuvant DC vaccination are equally effective

glioblastoma recommend a second course of radiotherapy in local recurrence to be considered, especially if there has been a long interval since prior irradiation and/or if there has been a good response to first-line treatment [23]. Additionally, a recent European review concluded that patients with a Karnofsky performance score (KPS) greater than $60 \%$, progression more than 6 months from time of surgery and a tumor size of up to $40 \mathrm{~mm}$ were the best candidates [17]. The ongoing Radiation Therapy Oncology Group (RTOG) 1205 trial is evaluating the benefit of reirradiation in a prospective and randomized setting (concurrent bevacizumab and re-irradiation versus bevacizumab alone as treatment for recurrent glioblastoma).

Due to the small number of prospective, randomized trials on relapsed HGG retrospective comparisons of competing salvage treatment strategies remain indispensable for clinical decision making to date. However, a confusing variety of potential prognostic factors, confounders and heterogeneous patient cohorts make benchmarking extremely difficult. In our opinion, simple, reliable and reproducible models classifying patients into homogeneous subgroups with similar intrinsic prognosis would be a way out of the dilemma. Of note, such models should ideally be based on predictors which are not related to a particular treatment ('pre-treatment variables'). The predictor "extent of resection", for instance, would not be appropriate as it implies repeated tumor resection. In 2012, the so-called HGG-Immuno RPA model was suggested. However, although being independent from treatment and perfectly meeting the criterion of simplicity, the model lacked successful validation with an independent patient cohort [7]. The present analysis now bridges this gap and certifies a simplified version of the model as fit for purpose. Simplification of the original model was mandatory because the predictor "mental status" had not been assessed in the majority of patients used for validation. This means that there are only three instead of four different prognostic classes and that patients aged $\geq 50$ years and diagnosed with glioblastoma are always in the worst prognostic group. KPS or mental status, do not have an additional meaning for their prognosis. We furthermore used the simplified model to compare two alternative salvage treatments (ReRT and ReOP + DCV) with each other. Although completely different neither of the two 
Table 1 Distribution of the predictors (ReOP + DCV cohort versus ReRT cohort)

\begin{tabular}{|c|c|c|}
\hline & $\mathrm{ReOP}+\mathrm{DCV}$ & ReRT \\
\hline $\mathrm{n}$ & 117 & 165 \\
\hline Era of salvage treatment & After 2003 & 1994-2012 \\
\hline \multicolumn{3}{|c|}{ Age at re-operation/re-irradiation } \\
\hline Median (range) (years) & n.a. & $52.2(17-81.1)$ \\
\hline$<50$ years & $62 / 117(53.0 \%)$ & $74 / 165(44.8 \%)$ \\
\hline$\geq 50$ years & $55 / 117(47.0 \%)$ & $91 / 165(55.2 \%)$ \\
\hline \multicolumn{3}{|l|}{ Histology } \\
\hline WHO grade III & $22 / 117(18.8 \%)$ & $30 / 165(18.2 \%)$ \\
\hline WHO grade IV & $95 / 117(81.2 \%)$ & $135 / 165(81.8 \%)$ \\
\hline \multicolumn{3}{|l|}{ KPI } \\
\hline Unknown & - & $1 / 165(0.6 \%)$ \\
\hline$<70$ & n.a. & $22 / 165(13.3 \%)$ \\
\hline $70-80$ & n.a. & $54 / 165(32.7 \%)$ \\
\hline$\geq 90$ & n.a. & $88 / 165(53.3 \%)$ \\
\hline \multicolumn{3}{|c|}{ RPA class (simplified model) } \\
\hline 1 & $11 / 117(9.4 \%)$ & $20 / 165(12.1 \%)$ \\
\hline 2 & $31 / 117(26.5 \%)$ & $39 / 165(23.6 \%)$ \\
\hline 3 & $75 / 117(64.1 \%)$ & $106 / 165(64.2 \%)$ \\
\hline
\end{tabular}

$R P A$ recursive partitioning analysis, KPI Karnofsky performance score, n.a. (information) not available

Table 2 6- and 12-month overall survival rates by prognostic score (ReOP + DVC cohort versus ReRT cohort)

\begin{tabular}{|c|c|c|c|c|}
\hline & $\begin{array}{l}6 \text { months- } \\
\text { OS }\end{array}$ & $(95 \% \mathrm{CI})$ & $\begin{array}{l}12 \text { months- } \\
\text { OS }\end{array}$ & $(95 \% \mathrm{CI})$ \\
\hline \multicolumn{5}{|c|}{ ReOP + DCV cohort $(n=117)$} \\
\hline Class I & 0.91 & $(0.75-1.00)$ & 0.73 & $(0.51-1.00)$ \\
\hline Class II & 0.90 & $(0.80-1.00)$ & 0.59 & $(0.43-0.80)$ \\
\hline $\begin{array}{c}\text { Class } \\
\text { III }\end{array}$ & 0.63 & $(0.53-0.75)$ & 0.25 & $(0.16-0.37)$ \\
\hline \multicolumn{5}{|c|}{ Re-RT cohort $(\mathrm{n}=165)$} \\
\hline Class I & 0.95 & $(0.85-1.00)$ & 0.72 & $(0.55-0.96)$ \\
\hline Class II & 0.72 & $(0.59-0.87)$ & 0.36 & $(0.23-0.55)$ \\
\hline $\begin{array}{c}\text { Class } \\
\text { III }\end{array}$ & 0.62 & $(0.53-0.72)$ & 0.23 & $(0.16-0.34)$ \\
\hline
\end{tabular}

$O S$ overall survival, $C I$ confidence interval

strategies was clearly superior to the other. Of note, in all prognostic classes we observed a tendency to slightly shorter survival times in the ReRT cohort, which was most evident in class II. However, this may be due to the fact that in the re-irradiated patients survival time was calculated from the start of re-irradiation, although a considerable number of these patients underwent prior cytoreductive re-surgery. In contrast, in the ReOP + DCV cohort, survival was calculated from the time-point of reoperation.

Naturally, it would be desirable to use the model for further studies assessing alternative salvage treatment approaches. Therefore, we are currently compiling a new dataset of relapsed high-grade glioma patients who received chemotherapy \pm bevacizumab as salvage treatment. We hope to be able to present first results soon.

\section{Conclusions}

In summary, the prognostic model's ability to discriminate patients with good, intermediate or bad prognosis was demonstrated in the cohort of re-irradiated patients. Being easy to handle and independent from the applied salvage treatment strategy, the simplified HGG-Immuno RPA model appears to be useful for therapeutic decision making. ReRT and ReOP + DVC seem to be similar with regards to efficacy. The choice between these two options for salvage treatment should mainly be based on the expected side effects.

Conflict of interest The authors indicated no potential conflicts of interest.

Ethical standards This manuscript is in accordance with the ethical standards laid down in the 1964 Declaration of Helsinki and its subsequent amendments.

\section{References}

1. Gallego O, Cuatrecasas M, Benavides M, Segura PP, Berrocal A, Erill N, Colomer A, Quintana MJ, Balana C, Gil M, Gallardo A, Murata P, Barnadas A (2014) Efficacy of erlotinib in patients with relapsed gliobastoma multiforme who expressed EGFRVIII and PTEN determined by immunohistochemistry. J Neurooncol 116(2):413-419. doi:10.1007/s11060-013-1316-y

2. Olson JJ, Nayak L, Ormond DR, Wen PY, Kalkanis SN, Committee ACJG (2014) The role of cytotoxic chemotherapy in the management of progressive glioblastoma: a systematic review and evidence-based clinical practice guideline. J Neurooncol 118(3):501-555. doi:10.1007/s11060-013-1338-5

3. Olson JJ, Nayak L, Ormond DR, Wen PY, Kalkanis SN, Ryken TC, Committee ACJG (2014) The role of targeted therapies in the management of progressive glioblastoma: a systematic review and evidence-based clinical practice guideline. J Neurooncol 118(3):557-599. doi:10.1007/s11060-013-1339-4

4. Quick J, Gessler F, Dutzmann S, Hattingen E, Harter PN, Weise LM, Franz K, Seifert V, Senft C (2014) Benefit of tumor resection for recurrent glioblastoma. J Neurooncol 117(2):365-372. doi:10.1007/s11060-014-1397-2

5. Ryken TC, Kalkanis SN, Buatti JM, Olson JJ, Committee ACJG (2014) The role of cytoreductive surgery in the management of progressive glioblastoma: a systematic review and evidencebased clinical practice guideline. J Neurooncol 118(3):479-488. doi:10.1007/s11060-013-1336-7 
6. Ryu S, Buatti JM, Morris A, Kalkanis SN, Ryken TC, Olson JJ, Committee ACJG (2014) The role of radiotherapy in the management of progressive glioblastoma: a systematic review and evidence-based clinical practice guideline. J Neurooncol 118(3):489-499. doi:10.1007/s11060-013-1337-6

7. De Vleeschouwer S, Ardon H, Van Calenbergh F, Sciot R, Wilms G, van Loon J, Goffin J, Van Gool S (2012) Stratification according to HGG-IMMUNO RPA model predicts outcome in a large group of patients with relapsed malignant glioma treated by adjuvant postoperative dendritic cell vaccination. Cancer Immunol Immunother 61(11):2105-2112. doi:10.1007/s00262-0121271-Z

8. Clark GM, Hilsenbeck SG, Ravdin PM, De Laurentiis M, Osborne CK (1994) Prognostic factors: rationale and methods of analysis and integration. Breast Cancer Res Treat 32(1):105-112

9. De Vleeschouwer S, Fieuws S, Rutkowski S, Van Calenbergh F, Van Loon J, Goffin J, Sciot R, Wilms G, Demaerel P, WarmuthMetz M, Soerensen N, Wolff JE, Wagner S, Kaempgen E, Van Gool SW (2008) Postoperative adjuvant dendritic cell-based immunotherapy in patients with relapsed glioblastoma multiforme. Clin Cancer Res 14(10):3098-3104. doi:10.1158/10780432.CCR-07-4875

10. Henke G, Paulsen F, Steinbach JP, Ganswindt U, Isijanov H, Kortmann RD, Bamberg M, Belka C (2009) Hypofractionated reirradiation for recurrent malignant glioma. Strahlenther Onkol 185(2):113-119. doi:10.1007/s00066-009-1969-9

11. Hundsberger T, Brugge D, Putora PM, Weder P, Weber J, Plasswilm L (2013) Re-irradiation with and without bevacizumab as salvage therapy for recurrent or progressive high-grade gliomas. J Neurooncol 112(1):133-139. doi:10.1007/s11060-013-1044-3

12. Scholtyssek F, Zwiener I, Schlamann A, Seidel C, Meixensberger J, Bauer M, Hoffmann KT, Combs SE, von Bueren AO, Kortmann RD, Muller K (2013) Reirradiation in progressive highgrade gliomas: outcome, role of concurrent chemotherapy, prognostic factors and validation of a new prognostic score with an independent patient cohort. Radiat Oncol 8(1):161. doi:10. 1186/1748-717X-8-161

13. Altman DG, Royston P (2000) What do we mean by validating a prognostic model? Stat Med 19(4):453-473
14. Royston P, Altman DG (2013) External validation of a Cox prognostic model: principles and methods. BMC Med Res Methodol 13:33. doi:10.1186/1471-2288-13-33

15. Schemper M, Smith TL (1996) A note on quantifying follow-up in studies of failure time. Control Clin Trials 17(4):343-346

16. Weller M, Pfister SM, Wick W, Hegi ME, Reifenberger G, Stupp R (2013) Molecular neuro-oncology in clinical practice: a new horizon. Lancet Oncol 14(9):e370-e379. doi:10.1016/S14702045(13)70168-2

17. Weller M, Cloughesy T, Perry JR, Wick W (2013) Standards of care for treatment of recurrent glioblastoma: are we there yet? Neuro-Oncol 15(1):4-27. doi:10.1093/neuonc/nos273

18. Niyazi M, Siefert A, Schwarz SB, Ganswindt U, Kreth FW, Tonn JC, Belka C (2011) Therapeutic options for recurrent malignant glioma. Radiother Oncol 98(1):1-14. doi:10.1016/j.radonc.2010. 11.006

19. Chaichana KL, Zadnik P, Weingart JD, Olivi A, Gallia GL, Blakeley J, Lim M, Brem H, Quinones-Hinojosa A (2013) Multiple resections for patients with glioblastoma: prolonging survival. J Neurosurg 118(4):812-820. doi:10.3171/2012.9.JNS1277

20. Sayegh ET, Oh T, Fakurnejad S, Bloch O, Parsa AT (2014) Vaccine therapies for patients with glioblastoma. J Neurooncol 119(3):531-546. doi:10.1007/s11060-014-1502-6

21. Shah AH, Bregy A, Heros DO, Komotar RJ, Goldberg J (2013) Dendritic cell vaccine for recurrent high-grade gliomas in pediatric and adult subjects: clinical trial protocol. Neurosurgery 73(5):863-867. doi:10.1227/NEU.0000000000000107

22. Wick W, Fricke H, Junge K, Kobyakov G, Martens T, Heese O, Wiestler B, Schliesser MG, von Deimling A, Pichler J, Vetlova E, Harting I, Debus J, Hartmann C, Kunz C, Platten M, Bendszus M, Combs SE (2014) A phase II, randomized, study of weekly APG101 + reirradiation versus reirradiation in progressive glioblastoma. Clin Cancer Res 20(24):6304-6313. doi:10.1158/ 1078-0432.CCR-14-0951-T

23. NCCN Guidelines Version 1 (2013) Anaplastic gliomas/ glioblastoma. http://www.ncen.org/professionals/physician_gls/ pdf/cns.pdf 